\title{
ARTICLES
}

\section{Antiferromagnetism of the half-filled Anderson lattice in one dimension}

\author{
Costas Papatriantafillou \\ Institute of Materials Science, NCSR, “Demokritos,"' 15310 Agia Paraskevi, Athens, Greece \\ Nicholas Kioussis and Sung Ha Park \\ Department of Physics, California State University Northridge, Northridge, California 91330-8268
}

(Received 4 May 1999)

\begin{abstract}
The ground-state properties of the symmetric periodic Anderson model in one dimension and at half filling have been studied using the local mean-field (LMF) method. We have calculated the ground-state energy, the local $f$ and conduction moment, the effective hybridization, the double occupancy, and the projected $f$ and conduction density of states. The LMF results are in good agreement with Monte Carlo results and with second-order perturbation theory in the fluctuations results, under the extreme conditions of the one dimensionality. The $f$ - and $c$-projected density of states show that there is a smooth change in the partial density of states with increasing $U$, from those of an uncorrelated, hybridized system to those expected for a strongly correlated system, in which the conduction band and the $f$ states are decoupled. [S0163-1829(99)08443-X]
\end{abstract}

\section{INTRODUCTION}

In recent years there has been considerable interest in the behavior of heavy fermion systems from both the experimental $^{1}$ and theoretical ${ }^{2}$ points of view. These systems usually contain light rare-earth or actinide elements and show a variety of unusual properties. At high temperatures, they behave as metals with weakly interacting magnetic moments. As the temperature is lowered, heavy-fermion systems exhibit different kinds of ground states: antiferromagnetic, superconducting, spin-density waves, charge-density waves, and Kondo state. ${ }^{3}$ Recently, another class of heavyfermion systems has emerged, the heavy-fermion semiconductors. ${ }^{4}$ The wide variety of ground states is associated with the partial delocalization of the $4 f$ or $5 f$ electrons and the cooperative hybridization of the $f$-electron ions with the non-f-band electrons.

The periodic Anderson model (PAM) is thought to describe the general behavior of heavy-fermions systems, and thus has been an object of great theoretical interest. ${ }^{5-13}$ The two-band PAM is a simplified parametric description of a conduction band interacting with a periodic lattice of localized electron states. There is hybridization between the localized and the extended band and there is a local (on-site) electron-electron interaction between electrons that occupy the spatially compact $f$ states on the same site. A variety of approximate and perturbative techniques, such as the Gutzwiller variational approach ${ }^{5}$ the slave boson technique, ${ }^{6}$ and the large-orbital-degeneracy $1 / N$ expansion ${ }^{7}$ have been applied to the periodic Anderson model. Numerical studies of finite PAM chains at half-filling (when the number of electrons is twice the number of sites) employing Lanczos diagonalization techniques for four-site chains, ${ }^{8,9}$ and Monte Carlo simulations, ${ }^{10,11}$ for 16 -site systems have provided physical insight into the ground-state properties. Steiner et al. have applied the second-order perturbation theory in the fluctuations around the mean-field result to the onedimensional PAM at half-filling and found good agreement with the Monte Carlo results. ${ }^{12}$ More recently, Guerrero and $\mathrm{Yu}^{13}$ have used the density-matrix-renormalization-group (DMRG) method $^{14}$ to calculate the charge gap, the spin gap, and the quasiparticle gap for lattices as large as 24 sites.

In this paper we investigate the ground-state properties of the one-dimensional symmetric nondegenarate PAM at halffilling in the local mean-field (LMF) approximation. Although fluctuation effects are expected to yield corrections to the mean-field results, we expect that the qualitative nature of the mean-field state to be maintained and that the meanfield treatment can reveal the basic properties of the system throughout the entire parameter space (including band filling and temperature) in contrast to other approaches, such as the Gutzwiller approximation, ${ }^{5}$ the large orbital $1 / N$ expansion, ${ }^{7}$ and various perturbative approaches, each making a different set of approximations, and thereby limiting their applicability to small regions of the parameter space. The results of our calculations for the ground-state properties, i.e., the total energy, the $f$-magnetic moment, the effective hybridization, and the $f$ - and $c$-projected density of states will be compared with those of Monte Carlo (MC) and DMRG calculations under the extreme conditions of the one dimensionality. The good agreement of our results with MC and DMRG results puts the LMF approximation on a firmer basis in two and three dimensions, where only a few results are available ${ }^{15}$ and other approaches may be limited (for example, there is a sign problem in the Monte Carlo calculations away from halffilling). Even though our approach is not an exact one, it has the advantage that the method becomes simple enough to generalize its application to the calculation of finitetemperature properties, it can be generalized to the case of higher-dimensional lattices, and it allows for the study of all 
regions in the parameter space.

The paper is organized as follows. In Sec. II we introduce the one-dimensional Anderson lattice and introduce the LMF technique involving the functional integral approach within the static approximation. ${ }^{16-18}$ We will discuss how the electronic and magnetic behavior of a given magnetic structure can be determined self-consistently by means of a Green'sfunctions approach which uses a renormalized perturbation expansion for the self-energy. ${ }^{19}$ In Sec. III we present and discuss our results for the ground-state properties, namely, the ground-state energy, the effective hybridization, the double occupancy, the average $f$ and $c$ local moment, and the conduction- and $f$-projected local density of states for the symmetric PAM at half-filling. We compare also the LMF results with the Monte Carlo and the second-order perturbation theory in the fluctuations mean-field results. We present our conclusions in Sec. IV.

\section{THE MODEL}

The Hamiltonian for the one-dimensional periodic Anderson Hamiltonian is

$$
\begin{aligned}
H= & -t \sum_{i \sigma}\left(c_{i \sigma}^{\dagger} c_{i+1 \sigma}+\text { H.c. }\right)+E_{c} \sum_{i \sigma} n_{i \sigma}^{c}+E_{f} \sum_{i \sigma} n_{i \sigma}^{f} \\
& +U \sum_{i} n_{i \uparrow}^{f} n_{i \downarrow}^{f}+V \sum_{i \sigma}\left(f_{i \sigma}^{\dagger} c_{i \sigma}+\text { H.c. }\right) .
\end{aligned}
$$

Here, $t$ is the nearest-neighbor hopping matrix element for the conduction electrons, $c_{i \sigma}^{\dagger}\left(c_{i \sigma}\right)$ and $f_{i \sigma}^{\dagger}\left(f_{i \sigma}\right)$ create (annihilate) Wannier electrons in $c$ - and $f$-like orbitals on site $i$ with spin $\sigma$, respectively, $E_{f}$ and $E_{c}$ are the energy levels of the bare localized $f$ state and the center of the conduction band, respectively, $U$ is the on-site Coulomb repulsion of the $f$ electrons, $V$ is the on-site hybridization matrix element between the local $f$ orbitals and the conduction band, and $n_{i \sigma}^{c}$ $\equiv c_{i \sigma}^{\dagger} c_{i \sigma}$ and $n_{i, \sigma}^{f} \equiv f_{i \sigma}^{\dagger} f_{i \sigma}$ are the number operators for the two bands, respectively. In this paper we use a simple nearest-neighbor tight-binding model for the conductionband dispersion, $\epsilon_{k}=-2 t \cos k$; we choose our energy scale so that the center of this unhybridized conduction band is at zero $\left(E_{c}=0\right)$. We also assume no direct $f-f$ hopping and purely on-site hybridization.

We employ the static approximation introduced originaly by Hubbard ${ }^{16}$ in the study of his model, and developed further by Lacour-Gayet and Cyrot $^{17}$ and Economou et al. ${ }^{18}$ properly extended for the Anderson Hamiltonian. The static approximation is a Hartree-Fock-type local mean-field one in which the many-body term in Eq. (1) is approximated as

$$
U n_{i \uparrow}^{f} n_{i \downarrow}^{f} \simeq \epsilon_{i \uparrow}^{f} n_{i \uparrow}^{f}+\epsilon_{i \downarrow}^{f} n_{i \downarrow}^{f}-(1 / U) \epsilon_{i \uparrow}^{f} \epsilon_{i \downarrow}^{f},
$$

where $\epsilon_{i \uparrow}^{f}, \epsilon_{i \downarrow}^{f}$ are the local effective potentials of the $f$-state energy levels. Consequently, the original Hamiltonian splits into two interdepending subsystems for the spin $\uparrow$ and spin $\downarrow$ electrons, described by the one-electron Hamiltonian $H_{\sigma}$ of the form:

$$
H_{\sigma}=H_{0, \sigma}+\sum_{i} \epsilon_{i, \sigma}^{f} n_{i, \sigma}^{f},
$$

where

$$
\begin{aligned}
H_{0, \sigma}= & -t \sum_{i}\left(c_{i \sigma}^{\dagger} c_{i+1 \sigma}+\text { H.c. }\right)+E_{c} \sum_{i} n_{i \sigma}^{c}+E_{f} \sum_{i} n_{i \sigma}^{f} \\
& +V \sum_{i}\left(f_{i \sigma}^{\dagger} c_{i \sigma}+\text { H.c. }\right) .
\end{aligned}
$$

The many-body problem posed by Eq. (1) is thus reduced to a self-consistency problem for the local potentials $\epsilon_{i, \uparrow}^{f}, \epsilon_{i, \downarrow}^{f}$ through the relations

$$
\begin{aligned}
\epsilon_{i, \uparrow}^{f} & =U\left\langle n_{i, \downarrow}^{f}\right\rangle, \\
\epsilon_{i, \downarrow}^{f} & =U\left\langle n_{i, \uparrow}^{f}\right\rangle,
\end{aligned}
$$

where the average number of $f$ electrons with spin $\sigma$ at site $i$ is

$$
\left\langle n_{i, \sigma}^{f}\right\rangle=\int^{E_{F}} \rho_{i, \sigma}^{f}(E) d E .
$$

Here, $\rho_{i, \sigma}^{f}(E)$ is the local $f$ partial density of states of spin $\sigma$, and $E_{F}$ is the Fermi energy.

The conduction $(c)$ - and $f$-projected local density of states $\rho_{i, \sigma}^{c}(E)$ and $\rho_{i, \sigma}^{f}(E)$ are evaluated from

$$
\begin{aligned}
& \rho_{i, \sigma}^{c}(E)=-(1 / \pi) \operatorname{Im}\left\langle c, i\left|\hat{G}_{\sigma}(E)\right| c, i\right\rangle, \\
& \rho_{i, \sigma}^{f}(E)=-(1 / \pi) \operatorname{Im}\left\langle f, i\left|\hat{G}_{\sigma}(E)\right| f, i\right\rangle,
\end{aligned}
$$

where $|f, i\rangle,|c, i\rangle$ are the $f$ band and $c$ band Wannier states on site $i$, respectively, and the Green's functions, $\hat{G}_{\sigma}(E)$, is

$$
\hat{G}_{\sigma}(E)=\left(E-\hat{H}_{\sigma}\right)^{-1} \text {. }
$$

The diagonal matrix elements of the $\hat{G}_{\sigma}(E)$ 's can be calculated using the Anderson's renomalized perturbation expantion (RPE) method, ${ }^{19}$ which in the case of the two-band one-dimensional PAM are given in terms of $2 \times 2$ matrices accounting for the $f$ and $c$ bands. The diagonal matrix elements are given by

$$
G_{i i, \sigma}=\left|\begin{array}{cc}
\left(E-E_{c}-2 T_{i i, \sigma}\right) & -V \\
-V & \left(E-E_{f}-\epsilon_{i, \sigma}^{f}\right)
\end{array}\right|^{-1},
$$

where the scattering matrix $\mathbf{T}_{i, \sigma}$ is calculated from the recurrence relation

$$
\mathbf{T}_{i \pm 1, \sigma}=\mathbf{t}\left(\mathbf{E}-\epsilon_{\mathbf{i}, \sigma}-\mathbf{T}_{i, \sigma}\right)^{-1} \mathbf{t} .
$$

Here, $\mathbf{T}_{i, \sigma}, \epsilon_{\mathbf{i}, \sigma}, \mathbf{t}$, and $\mathbf{E}$ are $2 \times 2$ matrices given by

$$
\begin{gathered}
\mathbf{T}_{i, \sigma}=\left(\begin{array}{cc}
T_{i i, \sigma} & 0 \\
0 & 0
\end{array}\right), \quad \epsilon_{\mathbf{i}, \sigma}=\left(\begin{array}{cc}
E_{c} & V \\
V & E_{f}+e_{i, \sigma}^{f}
\end{array}\right), \\
\mathbf{t}=\left(\begin{array}{ll}
t & 0 \\
0 & 0
\end{array}\right), \quad \mathbf{E}=\left(\begin{array}{cc}
E & 0 \\
0 & E
\end{array}\right) .
\end{gathered}
$$

The self-consistency scheme in Eqs. (5) and (6) involves the calculation of the $c$ and $f$ partial density of states in Eqs. (8) and (9), which in turn requires an assumption pertaining to the form of the spatial homogeneity of the ground state of the system. In other words, an assumption about the various 
forms of periodicity in the values of the local potentials $\left\{\epsilon_{i \uparrow}^{f}, \epsilon_{i \downarrow}^{f}\right\}$, appropriate for the ground states of the PAM and the magnetic ordering (or absence of it) that corresponds to it.

The two most simple magnetically ordered states are (i) a spatially homogeneous (ferromagnetic) state, i.e., $\epsilon_{i, \sigma}^{f}$ $=\epsilon_{\sigma}(\sigma=\uparrow$ or $\downarrow)$ for all lattice sites $i$. In this case, the recurrence relation turns into an equation for $T_{i i, \sigma}=T_{\sigma}$ of the form

$$
T_{\sigma}=\frac{t^{2}\left(E-E_{f}-\epsilon_{\sigma}\right)}{\left(E-E_{f}-\epsilon_{\sigma}\right)\left(E-E_{c}-T_{\sigma}\right)-V^{2}},
$$

which gives complex values for $T_{\sigma}$ for energies $E$ within the bands of the system and a corresponding imaginary part for $\hat{G}_{i i, \sigma}(E)$.

(ii) An antiferromagnetic state consisting of two interpenetrating sublattices + and - , with local effective potentials $\epsilon_{\sigma}^{f}=\epsilon_{\sigma}^{+}$or $\epsilon_{\sigma}^{f}=\epsilon_{\sigma}^{-}$, depending if site $i$ occupies the $\{ \pm\}$ sublattice, respectively, and $\sigma=\uparrow$ or $\downarrow$. Note that due to symmetry, $\epsilon_{\uparrow}^{+}=\epsilon_{\downarrow}^{-}$and $\epsilon_{\downarrow}^{+}=\epsilon_{\uparrow}^{-}$. The antiferromagnetic state is found to be energetically favored at half-filling. In this case, the recurrence relation yields the following system of equations for $T_{i i, \sigma}=T_{\sigma}^{+}$and $T_{i i, \sigma}=T_{\sigma}^{-}$corresponding to the two sublattices:

$$
\begin{aligned}
& T_{\sigma}^{+}=t^{2}\left[E-E_{c}-T_{\sigma}^{-}-V^{2}\left(E-E_{f}-\epsilon_{\sigma}^{-}\right)^{-1}\right]^{-1}, \\
& T_{\sigma}^{-}=t^{2}\left[E-E_{c}-T_{\sigma}^{+}-V^{2}\left(E-E_{f}-\epsilon_{\sigma}^{+}\right)^{-1}\right]^{-1},
\end{aligned}
$$

which gives complex values for $T_{\sigma}^{+}$and $T_{\sigma}^{-}$for energies within the bands of the system and corresponding imaginary part for $\hat{G}_{\sigma, i i}(E)$. The self-consistency scheme will then yield values for the $\epsilon_{\sigma}^{+}, \epsilon_{\sigma}^{-}$for spin $\sigma=\uparrow$ or $\downarrow$, which in turn determines fully the antiferromagnetic state of the system.

For each magnetically ordered state the self-consistency iterative scheme proceeds as follows. One starts with plausible initial values for the local potentials $\epsilon_{\uparrow}^{f}, \epsilon_{\downarrow}^{f}$, and calculates the Fermi energy for a given value of the number of electrons per site (band filling), $n$, from

$$
n=\int^{E_{F}} \rho(E) d E,
$$

where the total density of states is

$$
\rho(E)=\sum_{\sigma}\left[\rho_{\sigma}^{f}(E)+\rho_{\sigma}^{c}(E)\right] .
$$

Here, $\rho_{\sigma}^{f}(E)$ and $\rho_{\sigma}^{c}(E)$ are the $f$ and $c$ partial density of states calculated from Eqs. (8) and (9), respectively. The average number of $f$ electrons with $\sigma=\uparrow$ or $\downarrow$ is determined from Eq. (7), and new values for the $\epsilon_{\uparrow}^{f}, \epsilon_{\downarrow}^{f}$ are obtained from Eqs. (5) and (6). This procedure is repeated until it converges to the self-consistent values of $\left\{\epsilon_{\uparrow}^{f}, \epsilon_{\downarrow}^{f}\right\}$. The ground-state energy of a given magnetically ordered configuration is

$$
E_{G}=\int{ }^{E_{F}} E \rho(E) d E-(1 / U) \epsilon_{\uparrow}^{f} \epsilon_{\downarrow}^{f}
$$

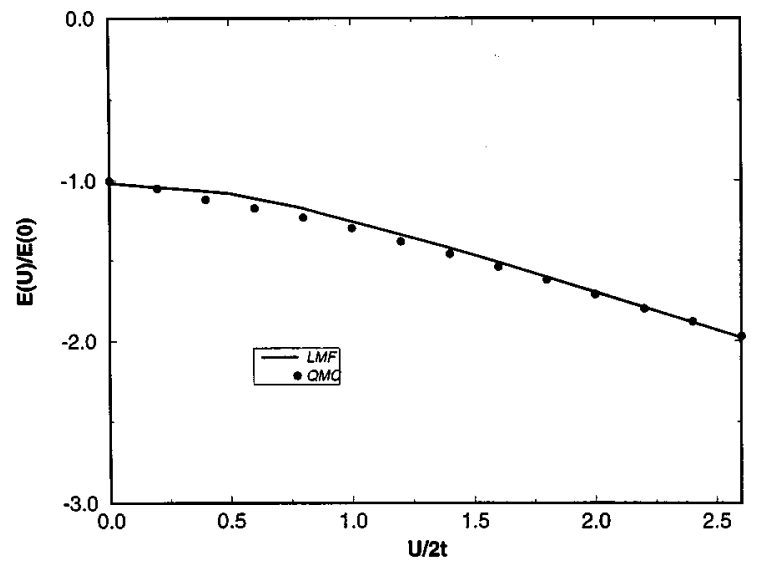

FIG. 1. Normalized ground-state energy $E(U) / E(O)$ plotted versus $U$ for the antiferromagnetic structure of the symmetric onedimensional PAM. The solid line is the results of the LMF method, and the closed circles are the Monte Carlo results (Ref. 10). Here, $t=0.5$ and $V=0.375$.

At half-filling $(N=2)$ we find that the antiferromagnetic state is the ground state of the system. The total magnetic moment $\mu_{i}$ on site $i$ is

$$
\mu_{i}=\mu_{i}^{f}+\mu_{i}^{c},
$$

where the $f$ and $c$ contributions to the local moment are

$$
\begin{aligned}
& \mu_{i}^{f}=\mu_{0}\left(\left\langle n_{i, \uparrow}^{f}\right\rangle-\left\langle n_{i, \downarrow}^{f}\right\rangle\right), \\
& \mu_{i}^{c}=\mu_{0}\left(\left\langle n_{i, \uparrow}^{c}\right\rangle-\left\langle n_{i, \downarrow}^{c}\right\rangle\right),
\end{aligned}
$$

respectively, and $\mu_{0}$ is the Bohr magneton constant.

\section{RESULTS AND DISCUSSION}

Even though in this work we present numerical results for the symmetric one-dimensional two-band PAM at halffilling, the LMF approach presented above can be used for arbitrary band filling, and thus it allows the study of the ( $U$, $V, E_{f}$ ) versus $n$ phase diagram for the one-dimensional PAM. The symmetric case corresponds to $E_{f}=-U / 2$. Although we have examined a range of different parameters, most of the results presented in this paper have been obtained with $V / t$ $=0.375$ and $\epsilon_{k}=-2 t \cos (k)$ with $t=0.5$, in order to be able to directly compare them with existing Monte Carlo results, ${ }^{10}$ results using the second-order perturbation theory in the fluctuations around the mean-field ${ }^{12}$ and DMRG [(Ref. 13)] results. All energies are in units of $2 t$.

In Fig. 1 we show the LMF results for the normalized ground-state energy, $E(U) / E(0)$, of the antiferromagnetic PAM (solid line) as a function of the Coulomb interaction $U$. Also shown in Fig. 1 are the Monte Carlo results. ${ }^{10}$ Note that the MC results are also in excellent agreement with those of Steiner et al. ${ }^{12}$ and the DMRG results. ${ }^{13}$ The excellent agreement of the LMF results with previous calculations in the entire $U$ range indicates that the MF approach yields accurate energetics. The LMF results smoothly crosses over from the small- $U$ expansion limit regime to the large- $U$ limit where a small- $V$ expansion is valid. Asymptotically, one obtains an energy of $-U / 2$, which is the contribution to the energy of the atomic level at $E_{f}$. 




FIG. 2. On-site square of the $f$ magnetic moment, $\left\langle m_{f}^{2}\right\rangle$, as a function of $U$ for the antiferromagnetic Anderson model (left and lower scale). The solid lines are the results of the LMF method, and the closed circles are the Monte Carlo results (Ref. 10). Here, $t$ $=0.5$ and $V=0.375$. Also shown is the double occupancy $D$ $=\left\langle n_{\uparrow}^{f} n_{\downarrow}^{f}\right\rangle$ for the antiferromagnetic state as a function of $U / 2 t$, for $V / 2 t=0.1$ and 0.375 (right and upper scale).

As a further corroboration of the validity of our approach, we compare our results for the square of the local $f$ moment,

$$
\left\langle m_{f}^{2}\right\rangle=\left\langle\left(n_{\uparrow}^{f}-n_{\downarrow}^{f}\right)^{2}\right\rangle=1-2\left\langle n_{\uparrow}^{f} n_{\downarrow}^{f}\right\rangle,
$$

with Monte Carlo results. ${ }^{10}$ In the LMF approach, charge and spin fluctuations are ignored, and $\left\langle n_{f \uparrow} n_{f \downarrow}\right\rangle=\left\langle n_{f \uparrow}\right\rangle\left\langle n_{f \downarrow}\right\rangle$. The square of the LMF $f$ moment, $\left\langle m_{f}^{2}\right\rangle$, is plotted versus $U / 2 t$ in Fig. 2 and compared with the Monte Carlo results (left and lower scale). For $U=0,\left\langle n_{f \uparrow}\right\rangle=\left\langle n_{f \downarrow}\right\rangle=\frac{1}{2}$, and $\left\langle m_{f}^{2}\right\rangle=\frac{1}{2}$. For large $U$, the double occupancy is reduced by the Coulomb repulsion and $\left\langle m_{f}^{2}\right\rangle$ approaches unity, implying that the instantaneous occupation of the spin-up orbital is strongly correlated with that of the spin-down orbital. The agreement between the MF and the Monte Carlo results is very good for $U / 2 t \geqslant 1(t=0.5)$. Note, that as expected, for small $U$ values $U / 2 t \leqslant 0.6$ the LMF approach gives a paramagnetic solution and the $\left\langle m_{f}^{2}\right\rangle=1 / 2$ is constant. Here, $U_{c}$ is the critical value of the Coulomb interaction for the onset of long-range antiferromagnetic order, which decreases as $V$ decreases. Both the LMF approach and the approach based on second-order perturbation theory in the fluctuations around the mean-field result ${ }^{12}$ understimate $\left\langle m_{f}^{2}\right\rangle$ in the intermediate-coupling regime. A measure of the effect of correlations is the number of double occupied $f$ sites $D=\left\langle n_{\uparrow}^{f} n_{\downarrow}^{f}\right\rangle$. In the MF approach $D=\left\langle n_{\uparrow}^{f}\right\rangle\left\langle n_{\downarrow}^{f}\right\rangle$. Also shown in Fig. 2 is the number of doubly occupied sites as a function of $U$, for two different values of hybridization $V / 2 t=0.375$ and 0.1 (right and upper scale). As expected, the double occupancy decreases with increasing $U$, indicating the increase in localization of the $f$ electrons $(D=0.25$ for the uncorrelated case of $U=0)$. For $V / 2 t$ $=0.375$ and for small values of $U\left(U<U_{c}=0.6\right)$ the system is in the paramagnetic phase and the double occupancy is constant $(0.25)$. As the hybridization decreases the double occupancy decreases and hence the local $f$ moment increases.

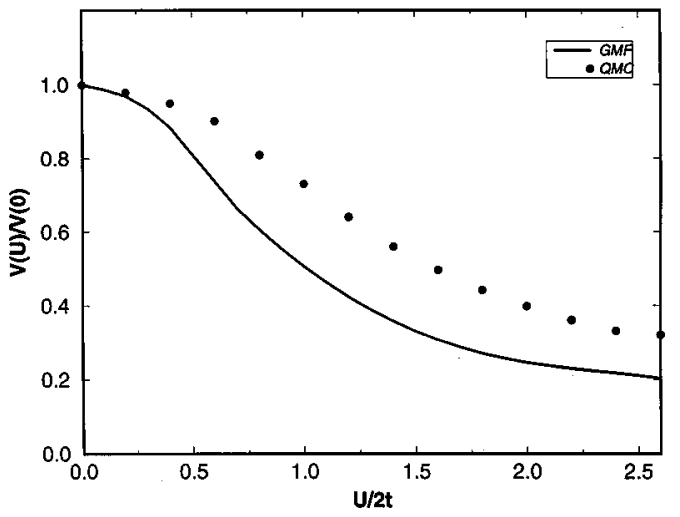

FIG. 3. Hybridization matrix element $V(U)=\left\langle f_{i \sigma}^{\dagger} c_{i \sigma}+c_{i \sigma}^{\dagger} f_{i \sigma}\right\rangle$ normalized to its $U=0$ value, $V(0)$, as a function of $U$ for the antiferromagnetic state for the same parameters as in Fig. 1. The solid line is the results of the LMF method and the closed circles are the Monte Carlo results.

For $V / 2 t=0.1$ the critical value of $U$ for the antiferromagnetic to paramagnetic transition decreases, and $D$ decreases more rapidly with $U$.

In the presence of $U$, the effective hybridization is reduced as a result of the Coulomb correlations. Blankenbecler et al. examined ${ }^{10}$ a normalized effective hybridization, defined by $\left\langle f_{i \sigma}^{\dagger} c_{i \sigma}+c_{i \sigma}^{\dagger} f_{i \sigma}\right\rangle /\left\langle f_{i \sigma}^{\dagger} c_{i \sigma}+c_{i \sigma}^{\dagger} f_{i \sigma}\right\rangle_{0}$, where $\left\langle f_{i \sigma}^{\dagger} c_{i \sigma}\right.$ $\left.+c_{i \sigma}^{\dagger} f_{i \sigma}\right\rangle_{0}$ is the hybridization when $U=0$. This effective hybridization is a measure of the $c-f$ charge fluctuations and can be directly obtained from the ground-state energy $E_{0}$ by the Feynman-Hellman relation

$$
\left\langle f_{i \sigma}^{\dagger} c_{i \sigma}+c_{i \sigma}^{\dagger} f_{i \sigma}\right\rangle=\frac{1}{2} \vartheta E_{0} / \vartheta V
$$

The LMF results (solid line) for the normalized effective hybridization is plotted versus $U$ in Fig. 3 and compared to the Monte Carlo results (closed circles). The effective hybridization decreases as $U$ increases resulting in the formation of local moments on the $f$ orbitals. The LMF results are in good agreement with those of the Monte Carlo calculations, being consistently underestimated by about $10 \%$ to $20 \%$.

The local $f$ moment $\mu_{f}$ and the conduction-electron moment $\mu_{c}$ are plotted versus $U$ for $V=0.375$ and 0.1 in Figs. 4(a) and 4(b), respectively. For $U<U_{c}$, the local $f$ moment $\mu_{f}$ vanishes (paramagnetic state) and $\mu_{f} \rightarrow 1$ for large $U$. The induced conduction-electron moment $\mu_{c}$ is small $(\sim 0.1)$ and is aligned antiferromagnetically with the local $f$ moment. As the hybridization decreases the local $f$ moment approaches its saturation value at a smaller $U$ value, due to the enhancement of the Ruderman-Kittel-Kasuya-Yosida (RKKY) exchange interaction $\left(J=\sum_{k}\left[V^{2} /\left(\epsilon_{k}-E_{f}\right)\right]\right)$, which is responsible for the long-range antiferromagnetic ordering.

In Figs. 5(a) and 5(b) we plot the $c$ - and $f$-projected LMF density of states, respectively, for the antiferromagnetic state for different values of $U / 2 t$ of 0,2 , and 4 . Note, the different energy scale for the $c$ - and $f$-projected density of states. For $U=0$, the upper and lower bands of the paramagnetic quasiparticle density of states are separated by the indirect gap

$$
E_{g}=2\left[\left(t^{2}+V^{2}\right)^{1 / 2}-t\right]
$$




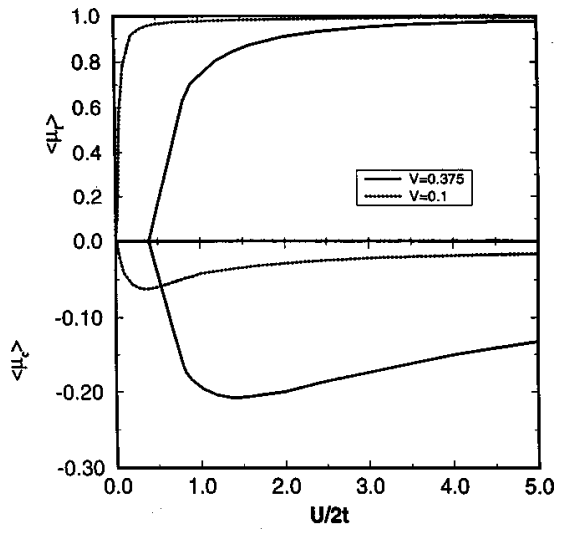

FIG. 4. (a) The local $f$ moment $\mu_{f}$ and (b) the conductionelectron moment $\mu_{c}$ for the antiferromagnetic state versus $U / 2 t$, for $V / 2 t=0.1$ and 0.375 .

For the symmetric case studied here, the bare $f$ energy scales with $U\left(E_{f}=-U / 2\right)$ so that all the symmetric case results presented as a function of $U$ are for systems with different values of $E_{f}$ as well. For small $U$, we expect the interacting lattice system to exhibit characteristics similar to this noninteracting limit. For the symmetric half-filled case considered in this work the Fermi energy is located in the middle of the gap $(\epsilon=0)$, and the system is an antiferromagnetic insulator. (a)

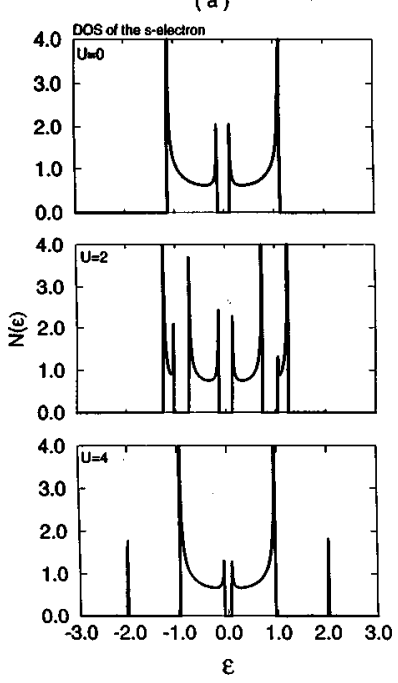

(b)

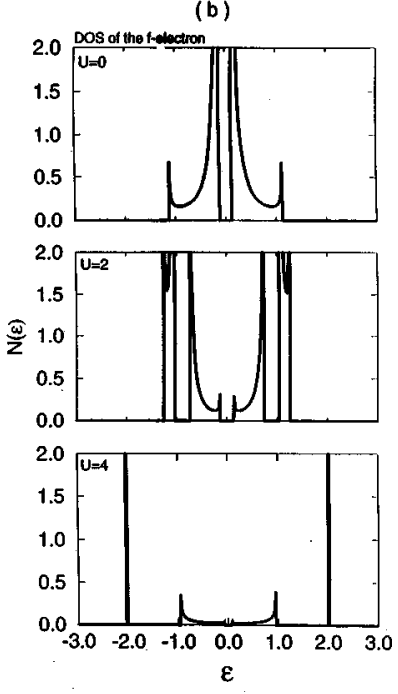

FIG. 5. (a) The conduction- and (b) the $f$-projected local density of states for the antiferromagnetic state for $U / 2 t=0,2$, and 4 . Here, $t=0.5$ and $V / 2 t=0.375$. The chemical potential is located at $\epsilon=0$.
One major set of roots is the nearly dispersionless and predominantly $f$ type of roots near the $E_{f}= \pm U / 2$. These correspond to the atomic satellites of the $f^{1} \rightarrow f^{2}$ and $f^{1} \rightarrow f^{0}$ excitations. Another major set of roots have predominantly $c$ character and a dispersion very close to $-\cos (k)$, the dispersion of the mean-field bands. An examination of the $f$ - and $c$ projected density of states with increasing $U$ shows that there is a smooth change in the partial density of states from those of an uncorrelated, hybridized system to those expected for a strongly correlated system, in which the conduction band and the $f$ states are decoupled, in agreement with the results of Steiner $e t a l .{ }^{12}$ This decoupling of the $f$ states from the conduction band can be measured more quantitatively by a reduction in the effective hybridization between the $f$ and $c$ states, in Fig. 3.

\section{CONCLUSIONS}

In this paper we have studied the ground-state properties of the one-dimensional symmetric $\left(E_{f}=-U / 2\right)$ periodic Anderson model at half-filling using the local mean-field approach. We find that at half-filling the ground state is antiferromagnetic. The results for the total energy, the on-site square of the $f$-magnetic moment, and the effective hybridization are in good agreement with Monte Carlo results ${ }^{10}$ and results using the second-order perturbation theory in the fluctuations. ${ }^{12}$ As expected with any mean-field treatment, we find a critical value of $U$ below which the state is paramagnetic. The $f$-and $c$-projected density of states show that there is a smooth change in the partial density of states with increasing $U$, from those of an uncorrelated, hybridized system to those expected for a strongly correlated system, in which the conduction band and the $f$ states are decoupled.

Eventhough the LMF approach is not an exact one, it has the advantage that the method becomes simple enough to generalize its application to the calculation of finitetemperature properties, it can be generalized to the case of higher-dimensional lattices, where only a few results are available, and it allows for the study of all regions in the parameter space. Future calculations will be aimed at studying the ground-state properties of the one-dimensional PAM when varying the poistion of the $f$-level $E_{f}$ and the band filling, thus constructing the phase diagram.

\section{ACKNOWLEDGMENTS}

This work was supported through the National Science Foundation under Grant No. DMR-9531005, the North Atlantic Treaty Organization under Grants Nos. HTECH.LG. 970619 and 941299, and the Parsons Foundation.
${ }^{1}$ For an experimental review, see G. R. Stewart, Rev. Mod. Phys. 56, 755 (1984).

${ }^{2}$ P. A. Lee, T. M. Rice, J. W. Serene, L. J. Sham, and J. W. Wilkins, Condens. Matter Phys. 12, 99 (1986).

${ }^{3}$ Z. Fisk, D. W. Hess, C. J. Pethick, D. Pines, J. L. Smith, J. D. Thompson, and J. O. Willis, Science 239, 33 (1988).

${ }^{4}$ G. Aeppli and Z. Fisk, Comments Condens. Matter Phys. 16, 155
(1992); A. Severing, J. D. Thompson, P. C. Canfield, Z. Fisk, and P. S. Riseborough, Phys. Rev. B 44, 6832 (1991).

${ }^{5}$ T. M. Rice and K. Ueda, Phys. Rev. B 34, 6420 (1986); B. H. Brandow, ibid. 33, 215 (1986).

${ }^{6}$ P. S. Riseborough, Phys. Rev. B 45, 13984 (1992); G. Kotliar and A. E. Ruckenstein, Phys. Rev. Lett. 57, 1362 (1986); A. Millis and P. A. Lee, Phys. Rev. B 35, 3394 (1987). 
${ }^{7}$ N. Read, D. M. Newns, and S. Doniach, Phys. Rev. B 30, 3841 (1984).

${ }^{8}$ R. Jullien and R. M. Martin, Phys. Rev. B 26, 6173 (1982).

${ }^{9}$ J. Callaway, J. W. Kim, and L. Tan, Phys. Rev. B 48, 11545 (1993).

${ }^{10}$ R. Blankenbecler, J. R. Fulco, W. Gill, and D. J. Scalapino, Phys. Rev. Lett. 58, 411 (1987).

${ }^{11}$ R. M. Fye, Phys. Rev. B 41, 2490 (1990).

${ }^{12}$ M. M. Steiner, R. C. Albers, D. J. Scalapino, and L. J. Sham, Phys. Rev. B 43, 1637 (1991).
${ }^{13}$ M. Guerrero and C. C. Yu, Phys. Rev. B 51, 10301 (1995).

${ }^{14}$ S. R. White, Phys. Rev. Lett. 68, 3487 (1992).

${ }^{15}$ M. Vekić, J. W. Cannon, D. J. Scalapino, R. T. Scalettar, and R. L. Sugar, Phys. Rev. Lett. 74, 2367 (1995).

${ }^{16}$ J. Hubbard, Proc. R. Soc. London, Ser. A 281, 401 (1964).

${ }^{17}$ P. Lacour-Gayet and M. Cyrot, J. Phys. C 7, 400 (1974).

${ }^{18}$ E. N. Economou, C. T. White, and R. R. DeMarco, Phys. Rev. B 18, 3946 (1977).

${ }^{19}$ C. Papatriantafillou, Phys. Rev. B 7, 5386 (1973). 